\title{
MAMMALS
}

\section{SASKATCHEWAN CHRISTMAS MAMMAL COUNT - 1999}

Compiled by Wayne C. Harris, Saskatchewan Environment and Resource Management, 350 Cheadle Street West, Swift Current, SK S9H 4G3

The number of mammal counts received this year is 94 , two less than in 1997, but seven more than received last year. The number of species reported was 37 , two less than both 1998 and 1997. The average number of species per count was 7.2 , the same as last year, which is below the long-term average.

The weather was mild and windy. Much of the south experienced snowfree conditions while further north the little snow cover present was crusted, making track identification difficult as evidenced by the low number of species and even one count reporting zero species.

No new species were reported this year. The Short-tailed Shrew at Fort Qu'Appelle was the only rarity. Population trends followed the pattern of previous years with the exception of White-tailed Deer whose numbers appeared to be below long term averages.

For weather, coverage and participants please refer to the Christmas Bird Count found elsewhere in this issue. In the tables, a numeral alone shows that the mammals were seen, while a letter following the number means that the number was inferred by the means defined below. A letter alone means that the species was present but estimating the numbers was impossible or that no attempt was made to do so. Symbols used in the tables are:
$T=$ tracks

$L=$ active lodge or hut

$D=$ dead animal found

$d=$ fresh diggings found

$S=$ smell or odour

$\mathrm{H}=$ heard

$0=$ present during the count period (December 16 to January 3) but not found on count day.

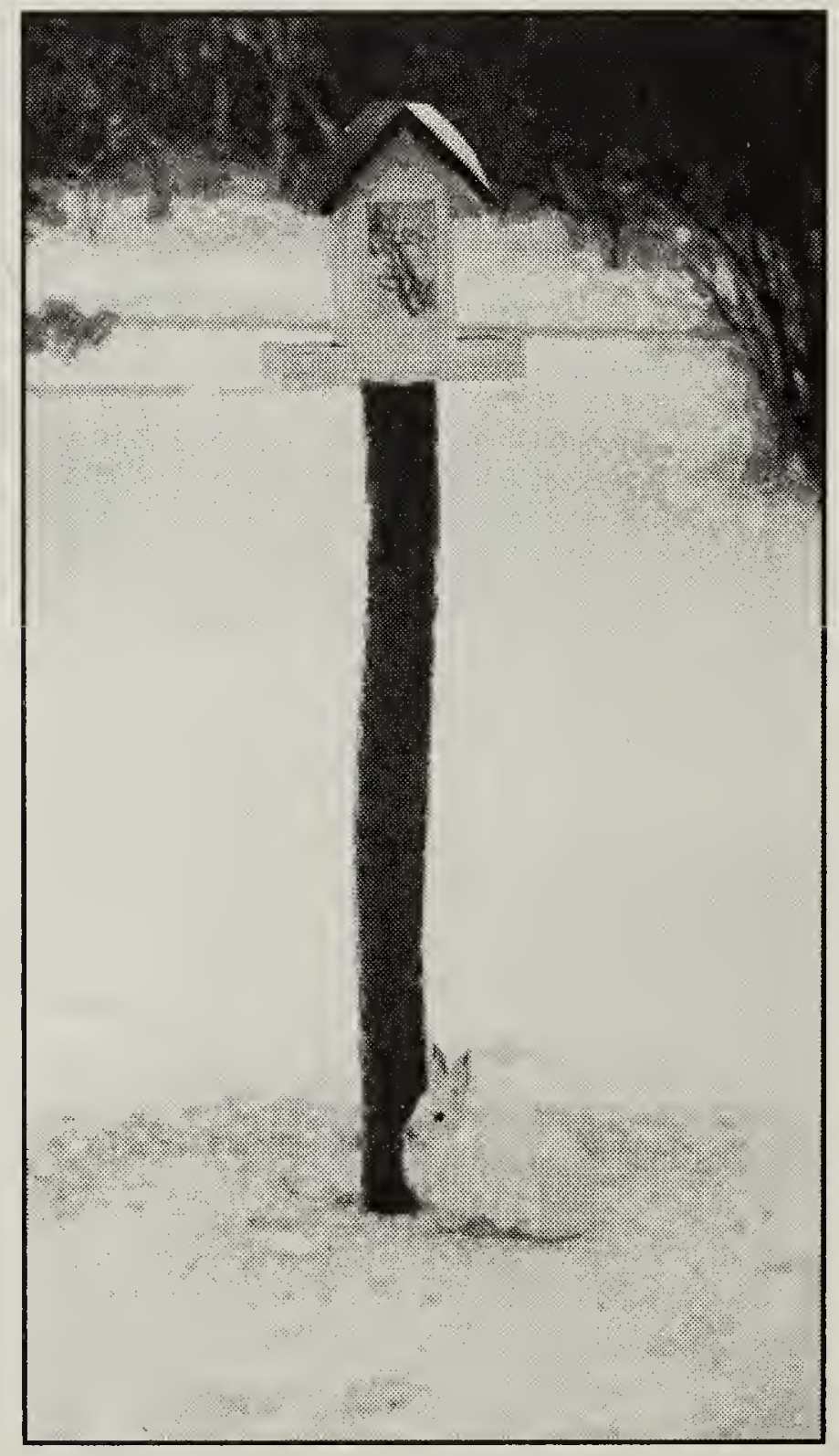

Snowshoe Hare at feeder - Birch Hills Don Weidt 
Table I-1: SASKATCHEWAN CHRISTMAS MAMMAL COUNTS

SPECIES

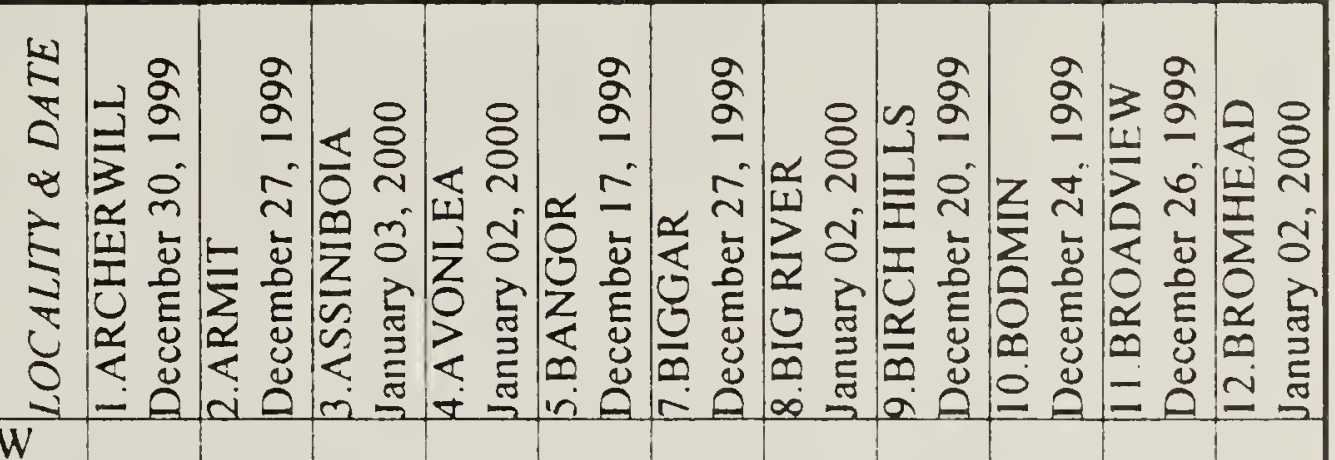

SHORT-TAILED SHREW MASKED SHREW

SHREW species

EASTERN COTTONTAIL

NUTTALL'S COTTONTAIL

SNOWSHOE HARE

WHITE-T. JACKRABBIT

RICH. GND. SQUIRREL

GREY SQUIRREL

FOX SQUIRREL

RED SQUIRREL

N. FLYING SQUIRREL

SQUIRREL species

BEAVER

DEER MOUSE

GAP. RED-BACKED VOLE

MUSKRAT

MEADOW VOLE

VOLE species

MOUSE species

NORWAY RAT

HOUSE MOUSE

PORCUPINE

COYOTE

WOLF

RED FOX

SWIFT FOX

RACOON

ERMINE

LONG-TAILED WEASEL

LEAST WEASEL

WEASEL species

MINK

BADGER

STRIPED SKUNK

RIVER OTTER

MULE DEER

WHITE-TAILED DEER

DEER species

MOOSE

ELK

PRONGHORN

TOTAL SPECIES

\begin{tabular}{|c|c|c|c|c|c|c|c|c|c|c|}
\hline 2 & $2 T$ & & & 14 & $1 \mathrm{~T}$ & & $2+24 \mathrm{~T}$ & $\mathrm{~T}$ & $2 T$ & \\
\hline $\mathrm{I}$ & & 11 & $10 \mathrm{~T}$ & & & & & & & 10 \\
\hline & & & & & & & & & & \\
\hline 5 & & & & & & & & & & \\
\hline & & & & & & & & & 2 & \\
\hline & 2 & & & 0 & & 1 & $1+1 T$ & 3. & & \\
\hline & & & & & & & & & & \\
\hline & & & 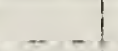 & & & & & & & \\
\hline & $2 \mathrm{~L}$ & & & & & & & & & \\
\hline & & & 0 & 12 & & & & & & \\
\hline & & & & & & & & 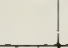 & & \\
\hline & $7 \mathrm{~L}$ & & $40 \mathrm{~L}$ & & & & & & & \\
\hline & & & & 6 & & & & & & \\
\hline & & & & & $4 \mathrm{~T}$ & & $1 T$ & & & \\
\hline & . & & & & & & $2 \mathrm{~T}$ & $\mathrm{~T}$ & $2 \mathrm{~T}$ & \\
\hline & & & & 1 & & & & & & \\
\hline & & & & 2 & & & & 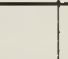 & & \\
\hline 2 & $2 \mathrm{~T}$ & 6 & & $1 T$ & & & & & & \\
\hline 3. & 1 & 9 & 1 & $5 \mathrm{H}$ & $2 \mathrm{~T}$ & $\mathrm{~T}$ & $2+6 \mathrm{~T}$ & $T$ & & 27 \\
\hline 1 & $1 \mathrm{~T}$ & & & & & & & & & \\
\hline 1 & & & 0 & & $1 \mathrm{~T}$ & $\mathrm{~T}$ & IT & & & 37 \\
\hline & & & & & & & & & & \\
\hline & & & & & . & & & - & & \\
\hline & & & & & & & $3 \mathrm{~T}$ & & & \\
\hline t & & & & & & & IT & & $1 \mathrm{Ti}$ & \\
\hline & & & & & & & $\mid \mathrm{TT}$ & & & \\
\hline & $1 \mathrm{~T}$ & & & & & & & 1 & & \\
\hline & & & & & & & & & $1 \mathrm{~T}$ & \\
\hline & & & & & & & IT & & & \\
\hline & & 3 & & & & & & & & \\
\hline & & & & & & & & & & \\
\hline & & 20 & & & 1 & & & & & \\
\hline & $3+50 \mathrm{~T}$ & 22 & 20 & 9 & $40 \mathrm{~T}$ & $\mathrm{~T}$ & $\mid 1+2 T$ & $\mathrm{~T}$ & $4 \mathrm{~T}$ & T \\
\hline & & & & & & & & & & \\
\hline 3 & $5 \mathrm{~T}$ & 1 & & & & & & & & \\
\hline & $10 \mathrm{~T}$ & & & & & & & & & \\
\hline & & 2 & & & & & ! & & & \\
\hline 9 & $11:$ & 8 & 6 & 10 & 6 & 41 & $11 !$ & 6 & 6 & 5 \\
\hline
\end{tabular}




\begin{tabular}{|c|c|c|c|c|c|c|c|c|c|c|c|}
\hline 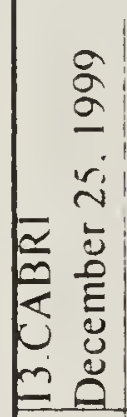 & 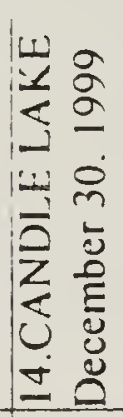 & 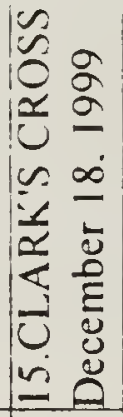 & 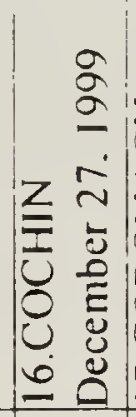 & 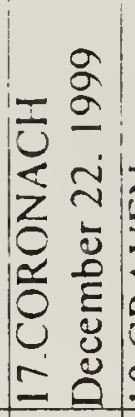 & 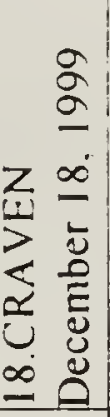 & 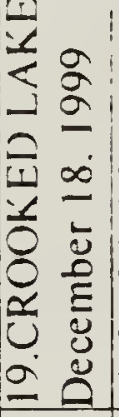 & 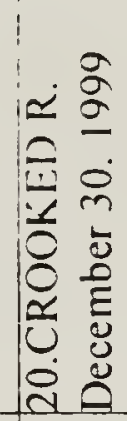 & 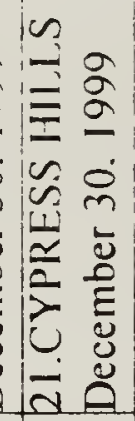 & 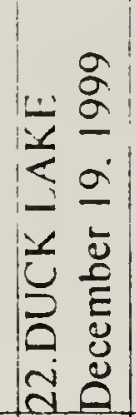 & 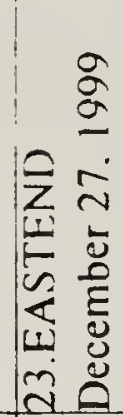 & $\left.\right|_{0}$ \\
\hline & & & & & & & & & & & SHORT-TAILED SHREW \\
\hline & & & & & & & & & & & MASKED SHREW \\
\hline & & & & & & $\mathrm{T}$ & $\Gamma$ & & & & SHREW species \\
\hline & & & & & & & & & & & EASTERN COTTONTAIL \\
\hline & & & & $5 \mathrm{~T}$ & & & & & & $1 \mathrm{~T}$ & TINUTTALLS COTTONTAIL \\
\hline & $8 \mathrm{~T}$ & $20 \mathrm{~T}$ & $2+2 T$ & & $2 \mathrm{~T}$ & & 1 & & & & SNOWSHOE HARE \\
\hline $3 \mathrm{~T}$ & & $1+1 T$ & 1 & 2 & $20 \mathrm{~T}$ & & 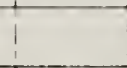 & & & & WHITE-T. JACKRABBIT \\
\hline & & & & $\mathrm{I}$ & & & 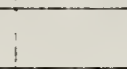 & & & & OICH. GND. SQUIRREL \\
\hline & & & & & 4 & & 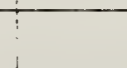 & & & & GREY SQUIRREL \\
\hline & & & & & 5 & & & & 1 & & FOX SQUIRREL \\
\hline & $3+9 \mathrm{~T}$ & IT & 1 & 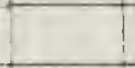 & $1 \mathrm{~T}$ & 5 & 5 & 28 & & & RED SQUIRREL \\
\hline & & & & & & & 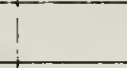 & & & & N. FLYING SQUIRREL \\
\hline & & & & & & & & & & & SQUIRREL species \\
\hline & & & & & 2 & $1 \mathrm{~L}$ & & & & $\mathrm{~L} 2$ & 2. BEAVER \\
\hline & & & $10 \mathrm{~T}$ & 2 & & $\mathrm{~T}$ & $\Gamma$ & & & $2 \mathrm{~T}$ & TIDEER MOUSE \\
\hline & & & & & & & & & & & GAP. RED-BACKED VOLE \\
\hline & & IT & & 1 & 3 & I & I & & & $5 \mathrm{~L}$ & LMUSKRAT \\
\hline & & & & 3 & 2 & $\mathrm{~T}$ & $\Gamma$ & I & I & IT & TIMEADOW VOLE \\
\hline & & $2+2 \mathrm{~T}$ & & & $2 \mathrm{~T}$ & & & & & & VOLE species \\
\hline & $2 \mathrm{~T}$ & $3 \mathrm{~T}$ & & $2 \mathrm{~T}$ & $17 \mathrm{~T}$ & & & & & & MOUSE species \\
\hline & & & & & & & & & & & NORWAY RAT \\
\hline & & & & & & & & 1 & & & HOUSE MOUSE \\
\hline & & 2 & $1 \mathrm{~T}$ & $3 !$ & 1 & & & & & & PORCUPINE \\
\hline $3 \mathrm{~T}$ & $1+2 T$ & & $3+3 T$ & 6 & 4 & 2 & () & () & 6 & & 2 COYOTE \\
\hline & & & & & & 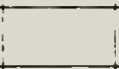 & 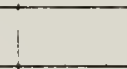 & & & & $0 \mathrm{WOLF}$ \\
\hline $\mathrm{I}$ & & IT & $1+2 \mathrm{~T}$ & $1+2 T$ & $2 \mathrm{~T}$ & $\mathrm{~T}$ & 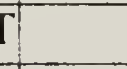 & & & IT & RED FOX \\
\hline & & & & & & & & & & & SWIFT FOX \\
\hline & & & & & & & & & & 0 & RACOON \\
\hline & & & & & & & & & & & ERMINE \\
\hline & & & & $\mathrm{IT}$ & $\mathrm{T}$ & & & & & & LONG-TAILED WEASEL \\
\hline & & & & & $1 \mathrm{~T}$ & & & & & & LEAST WEASEL \\
\hline & & I & I & IT & & & & & & & WEASEL species \\
\hline & 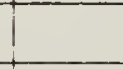 & & & & 1 & 3 & 3 & & & & MINK \\
\hline & & & & Id & & & & & & & BADGER \\
\hline $1 \mathrm{~S}$ & & IS & $1 \mathrm{~S}$ & $2 \mathrm{~S}$ & & & 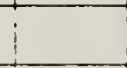 & & & & ISTRIPED SKUNK \\
\hline & & & & & & & & & & & RIVER OTTER \\
\hline 18 & & & 4 & 92 & & & & 18 & & 19 & 9 MULE DEER \\
\hline & $11+10 T$ & & $84+15 \mathrm{~T}$ & 36 & 16 & $\mathrm{~T}$ & 11 & $4 \overline{6}$ & & & 4) WHITE-TAILED DEER \\
\hline & & $7 \mathrm{~T}$ & & & $10 \mathrm{~T}$ & & & & $i$ & & DEER species \\
\hline & IT & & & & & & & 4 & 4 & & MOOSE \\
\hline & & & & & & & & & & & D. ELK \\
\hline & & & & 32 & & & & & & & P PRONGHORN \\
\hline 5 & 5 & 12 & 10 & 15 & 17 & 11 & & 2 & 0 & 14 & 4) TOTAL SPECIES \\
\hline
\end{tabular}


SPECIES

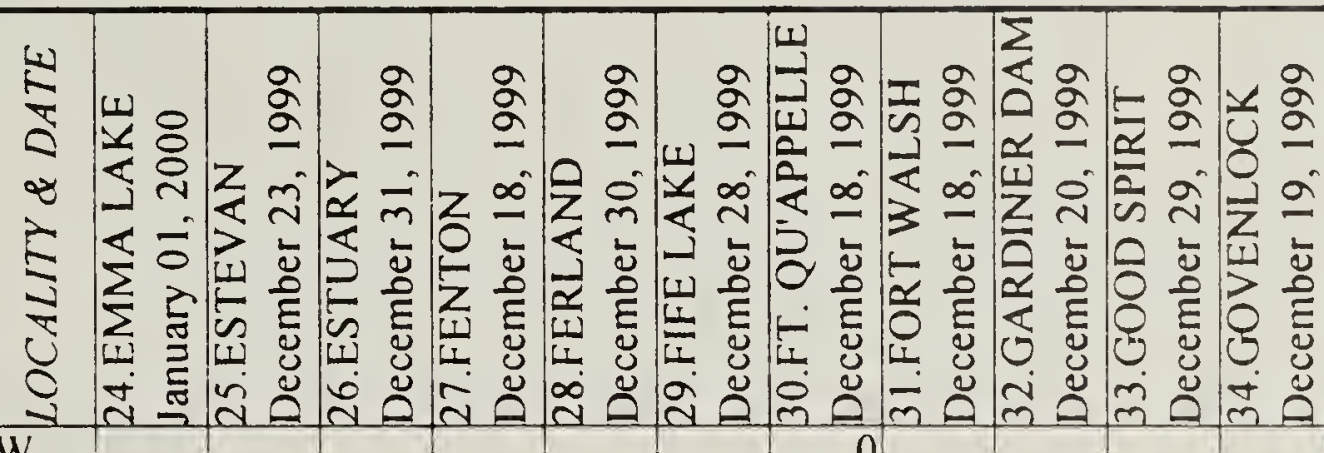
SHORT-TAILED SHREW MASKED SHREW

SHREW species

EASTERN COTTONTAIL

NUTTALL'S COT TONTAIL

SNOWSHOE HARE

WHITE-T. JACKRABBIT

RICH. GND. SQUIRREL

GREY SQUIRREL

FUX SQUIRREL

RED SQUIRREL

N. FLYING SQUIRREL

SQUIRREL species

BEAVER

DEER MUUSE

GAP. RED-BACKED VOLE

MUSKRAT

MEADOW VOLE

NOLE species

MOUSE Species

NORWAY RAT

HOUSE MOUSE

PORCUPINE

COYOTE

WOLF

RED FOX

SWIFT FOX

RACOON

ERMINE

LONG-TAILED WEASEL

LEAST WEASEL

WEASEL species

MINK

BADGER

STRIPED SKUNK

RIVER OTTER

MULE DEER

WHITE-TAILED DEER

DEER species

MOOSE

ELK

PRONGHORN

TOTAL SPECIES

\begin{tabular}{|c|c|c|c|c|c|c|c|c|c|c|}
\hline & & & & & & & & & \\
\hline & & & $2 \mathrm{~T}$ & & & & & & & \\
\hline & $5 T$ & & & & & & & & & \\
\hline & & 1 & & & & & $6 \mathrm{~T}$ & 4 & & \\
\hline 0 & $2 T$ & & $9 \mathrm{~T}$ & & & 1 & & $1 \mathrm{~T}$ & & \\
\hline & & & & & & & & $4+2 T$ & IT & \\
\hline & & & & & & & 1 & & & \\
\hline & & & & & & & & & & \\
\hline & 3 & & & 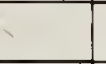 & & & & & & \\
\hline 5 & & & $1 \mathrm{H}+3$ & & & 10 & $7+2 T$ & & $1 \mathrm{~T}$ & \\
\hline 0 & & & & & & & & & & \\
\hline & & & & & & 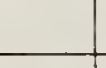 & & & + & \\
\hline & $2 \mathrm{~L}$ & & $1 \mathrm{~L}$ & & & 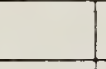 & & & $1 \mathrm{~L}$ & \\
\hline & $1 \mathrm{~T}$ & & & & & 2 & $3 T$ & & & \\
\hline & & & & & & 2 & & & & \\
\hline & $7 \mathrm{~L}$ & & & & 2 & 2 & & $12 \mathrm{~L}$ & $9 \mathrm{~L}$ & \\
\hline & ID & & & & & & 5 & 1 & & \\
\hline & & & & & & 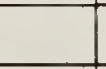 & $1 \mathrm{~T}$ & $2 \mathrm{~T}$ & $1 \mathrm{~T}$ & \\
\hline & & & $7 \mathrm{~T}$ & & & - & & $1 \mathrm{~T}$ & & \\
\hline & & & & & & & & & & \\
\hline & & & & & & 0 & & & & \\
\hline & & 1 & & & & 0 & $2 \mathrm{~T}$ & $4 \mathrm{~T}$ & $3 \mathrm{~T}$ & 37 \\
\hline & $3 \mathrm{~T}$ & 3 & $2+7 \mathrm{~T}$ & $4 \mathrm{H}$ & & 0 & 4 & 11 & 3 & \\
\hline & & & & & & 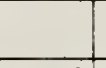 & & & & \\
\hline & $\mathrm{IT}_{3}$ & & & & & 0 & & $1+1 \mathrm{~T}$ & & \\
\hline & & & & & & & & & & 21 \\
\hline & & & & & & 0 & & & & \\
\hline & & & & & & & & & & \\
\hline & & & $2 \mathrm{~T}$ & & & & & & & \\
\hline & & & & & & & & & & \\
\hline & $1 \mathrm{~T}$ & & & & & & & & & \\
\hline & $1 \mathrm{~T}$ & & & & & I & & & & \\
\hline & & & & & & & & & & 10 \\
\hline & & & & & & & & & & \\
\hline & & & & & & & & & & \\
\hline & & 1 & & & & 0 & 15 & 141 & & 146 \\
\hline & $10 \mathrm{~T}$ & 19 & I0T & & & 0 & 44 & 29 & 7 & 25 \\
\hline & & & & & & & & & & \\
\hline & & & & & & & & & $1 \mathrm{~T}$ & \\
\hline & & & & & & & 1 & & & \\
\hline & & & & & & & 18 & & & 482 \\
\hline 3 & 12 & 5 & 8 & 1 & 1 & 14 & 12 & 111 & 9 & 8 \\
\hline
\end{tabular}




\begin{tabular}{|c|c|c|c|c|c|c|c|c|c|c|c|}
\hline 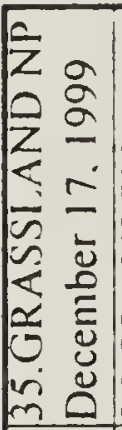 & 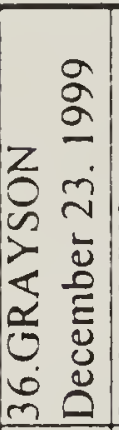 & 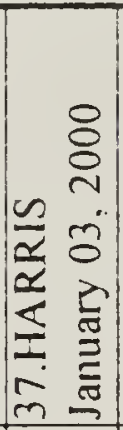 & 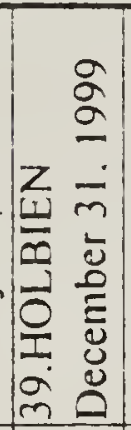 & 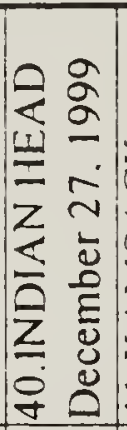 & 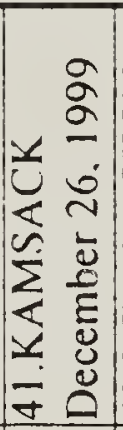 & 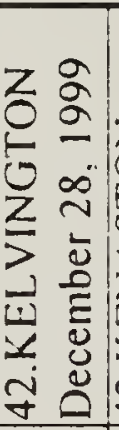 & 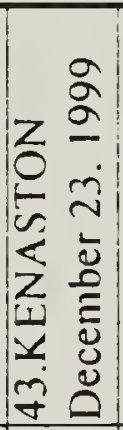 & 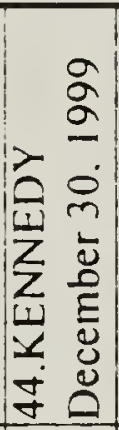 & 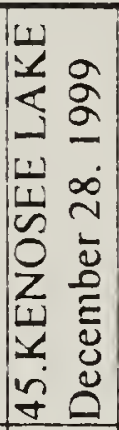 & 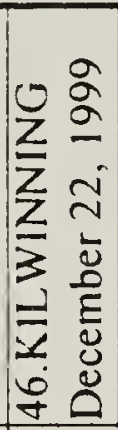 & 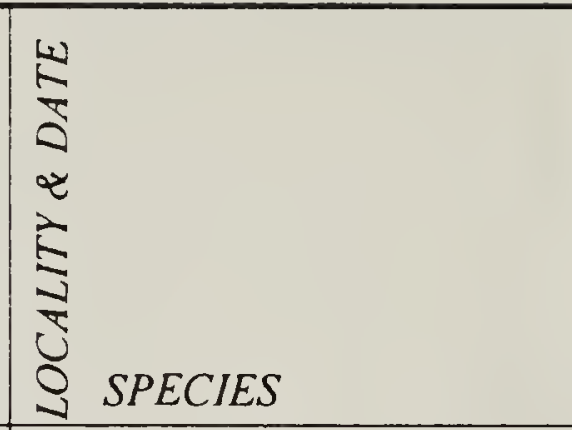 \\
\hline & & & & & & & & & & & SHORT-TAILED SHREW \\
\hline & & & & & & & & & & & MASKED SHREW \\
\hline & & & & & & & $12 \mathrm{~T}$ & & & & SHREW species \\
\hline & & & & & & & & & & & EASTERN COTTONTAIL \\
\hline & & & & & & & & & & & NUTTALL'S COTTONTAIL \\
\hline & & $6 \mathrm{~T}$ & & 2 & & I0T & $\mathrm{T}$ & $1 \mathrm{~T}$ & & & SNOWSHOE HARE \\
\hline & $1 \mathrm{~T}$ & $8 \mathrm{~T}$ & 3 & 3 & & & $T$ & & & & WHITE-T. JACKRABBIT \\
\hline & & & & & & & & & & & RICH. GND. SQUIRREL \\
\hline & & & & & & & & & & & GREY SQUIRREL \\
\hline & & & & & & & & & & & FOX SQUIRREL \\
\hline & 1 & 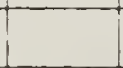 & & 21 & & & & & $5+3 \mathrm{H}$ & & RED SQUIRREL \\
\hline & & & & & & & & & & & N. FLYING SQUIRREL \\
\hline & & & & & & & & & & & SQUIRREL species \\
\hline & 1 & trat & & & & & & $2 \mathrm{~L}$ & $16 \mathrm{~L}$ & & BEAVER \\
\hline & & & & & & & $\mathrm{T}$ & & & & DEER MUUSE \\
\hline & & & & & & & & & & & GAP. RED-BACKED VOLE \\
\hline & & & & & & & & $29 \mathrm{~L}$ & $37 \mathrm{~L}$ & & MUSKRAT \\
\hline & 3 & 3 & & & & & $\mathrm{~T}$ & & & & MEADOW VOLE \\
\hline & & $3 \mathrm{~T}$ & & & & & & & & & VOLE species \\
\hline & $2 T$ & & 1 & ti & & & & & & & MOUSE species \\
\hline & & & & & & & & & & & NORWAY RAT \\
\hline & & & & & & & & & & & HOUSE MOUSE \\
\hline & & & & 1 & 1 & $1 \mathrm{~T}$ & & & & & PORCUPINE \\
\hline & $2 \mathrm{~T}$ & & 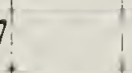 & 13 & $1 \mathrm{H}$ & 2 & 127 & $3 \mathrm{~T}$ & $\mathrm{I}+2 \mathrm{H}$ & & COYOTE \\
\hline & & & & & & & & & & & IWOLF \\
\hline & 1 & $4 \mathrm{~T}$ & & 1 & & & $12 \mathrm{~T}$ & & & & RED FOX \\
\hline & & & & & & & & & & & SWIFT FOX \\
\hline & & & & & & & $\mathrm{T}$ & $1 \mathrm{~T}$ & & & RACOON \\
\hline & & & & & & & & & & & ERMINE \\
\hline & & & $\mathrm{T}$ & ti & & & $\mathrm{T}$ & & & & LONG-TAILED WEASEL \\
\hline & & & & & & & & & & & LEAST WEASEL \\
\hline & $2 \mathrm{~T}$ & & & & & & & & & & WEASEL species \\
\hline & 3 & & & & & & $\mathrm{~T}$ & & & & MINK \\
\hline & & & & & & & T & & & & BADGER \\
\hline & & $1 \mathrm{~S}$ & & & 2 & & $\mathrm{~T}$ & & & & ISTRIPED SKUNK \\
\hline & & & & & & & & & & & RIVER OTTER \\
\hline 11 & & 44 & & & & & $T$ & & & & MULE DEER \\
\hline 56 & 1 & 34 & & 13 & $\overrightarrow{72}$ & $20 \mathrm{~T}^{7}$ & $\overrightarrow{\mathrm{T}}$ & 2 & 26 & & WHITE-TAILED DEER \\
\hline & & & & & & & & & & & DEER species \\
\hline & & & & & 2 & & & & & & MOOSE \\
\hline & & & & & T & & & & & & ELK \\
\hline 126 & & & & & & & & & & & PRONGHORN \\
\hline 5 & 10 & 8 & 3 & 7 & 6 & 4 & 14 & 6 & ti & & TOTAL SPECIES \\
\hline
\end{tabular}


Table I-5: SASKATCHEWAN CHRISTMAS MAMMAL COUNTS

SPECIES

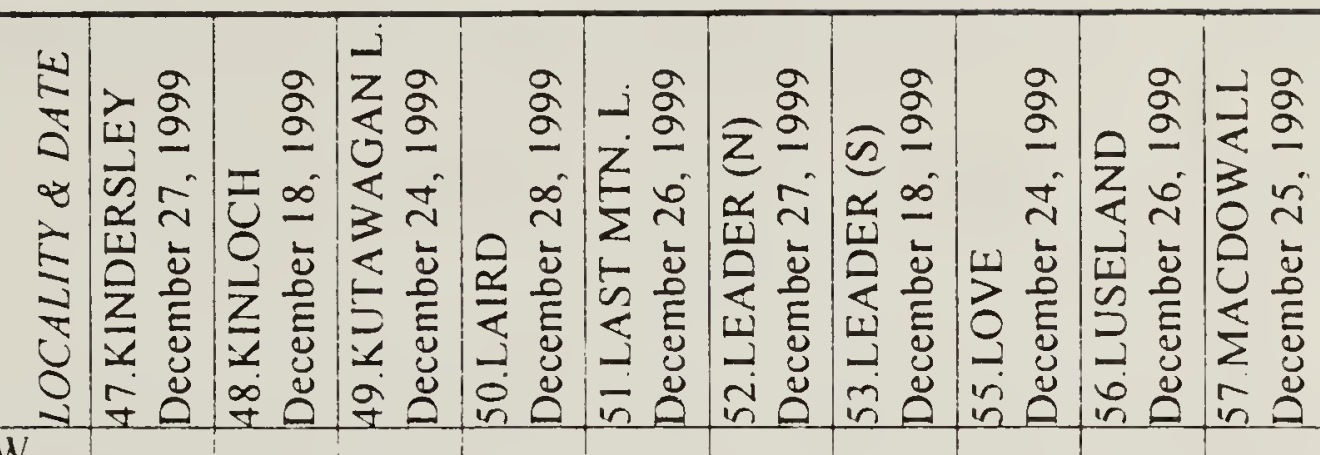
SHORT-TAILED SHREW MASKED SHREW

SHREW species

EASTERN COTTONTAIL

NUTTALL'S COTTONTAIL SNOWSHOE HARE WHITE-T. JACKRABBIT RICH. GND. SQUIRREL GREY SQUIRREL FOX SQUIRREL RED SQUIRREL

N. FLYING SQUIRREL SQUIRREL species BEAVER DEER MOUSE (IAP. RED-BACKED VOLE MUSKRAT MEADOW VOLE VOLE species MOUSE species NORWAY RAT HOUSE MOUSE PORCUPINE COYOTE WOLF RED FOX SWIFT FOX RACOON ERMINE LONG-TAILED WEASEL LEAST WEASEL WEASEL species MINK BADGER STRIPED SKUNK RIVER OTTER MULE DEER WHITE-TAILED DEER DEER species MOOSE ELK PRONGHORN

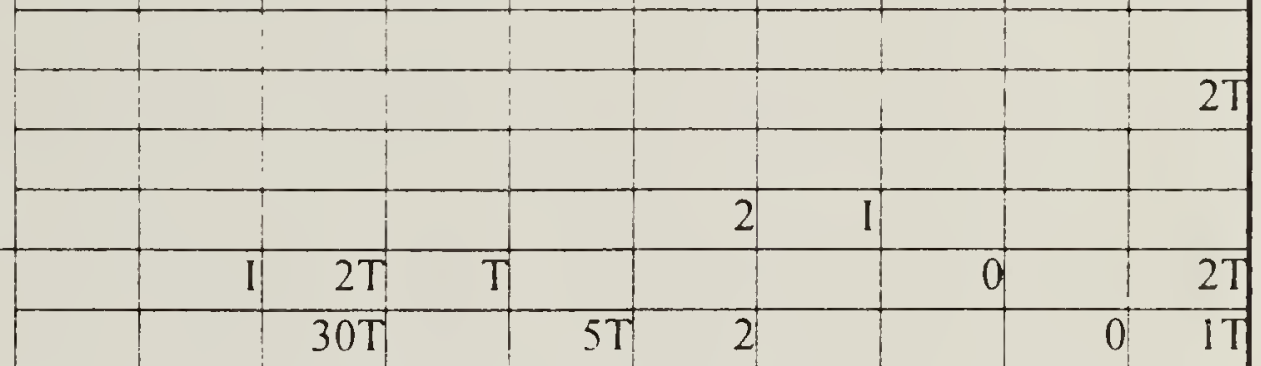

\begin{tabular}{|r|r|r|r|r|r|r|r|r|r|}
\hline & & $30 \mathrm{~T}$ & & $5 \mathrm{~T}$ & 2 & & & 0 & $1 \mathrm{~T}$ \\
\hline & & & & & & & & & \\
\hline & & & & & & & & & \\
\hline & $5 \mathrm{~T}$ & & & & & & 10 & \\
\hline & & & & & & & 0 & \\
\hline & & & & & & & & & \\
\hline & $2 \mathrm{~L}$ & & & & 1 & & & & 4 \\
\hline
\end{tabular}
TOTAL SPECIES

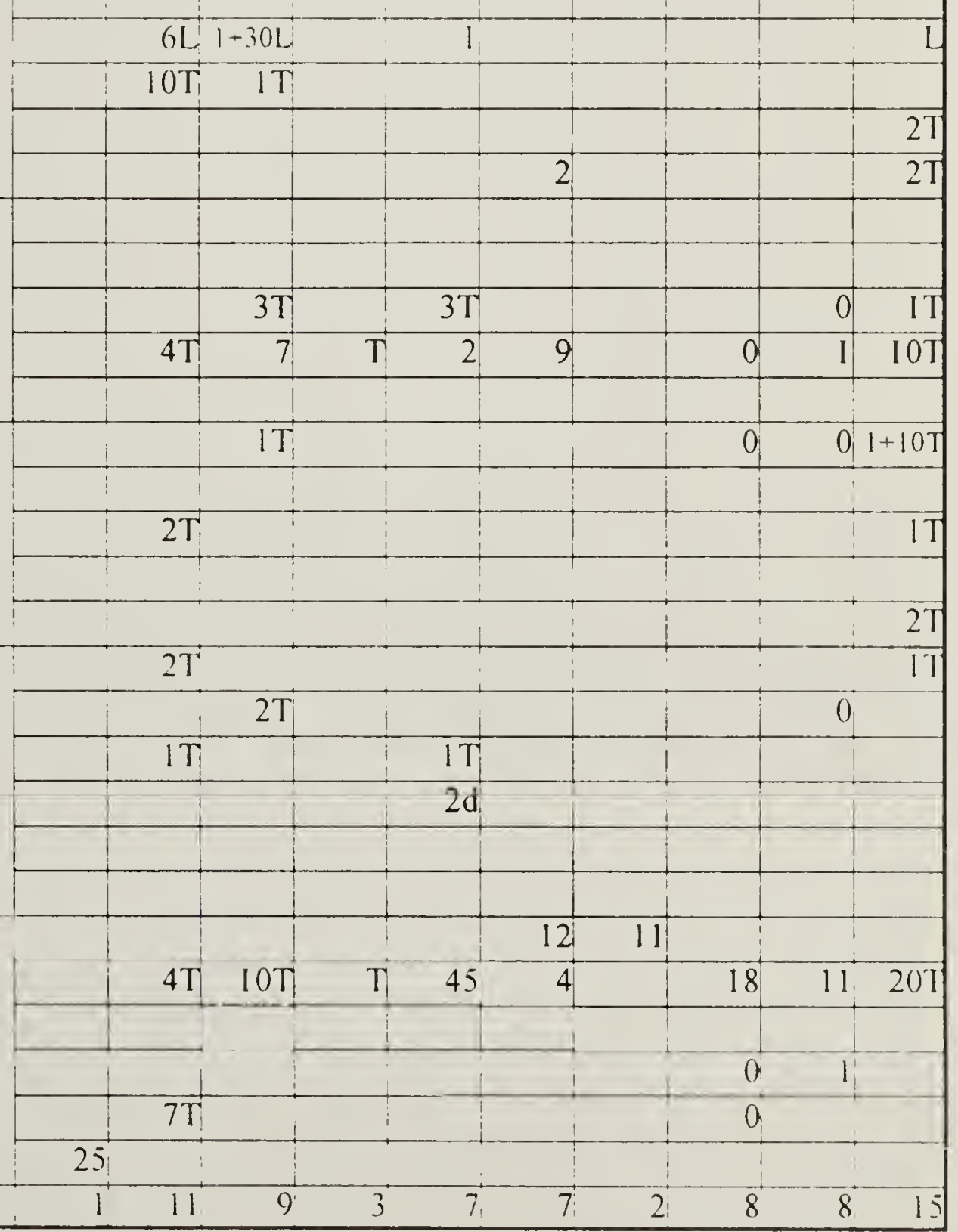




\begin{tabular}{|c|c|c|c|c|c|c|c|c|c|c|}
\hline 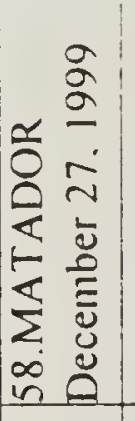 & 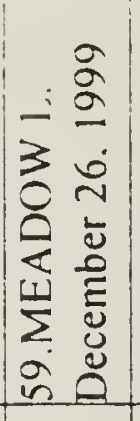 & 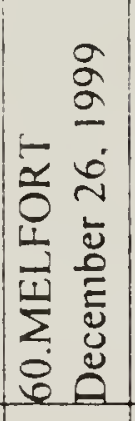 & 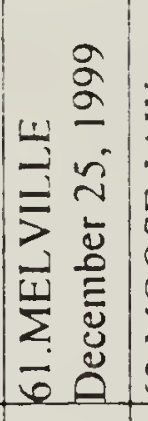 & 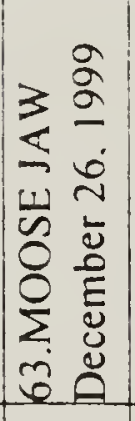 & 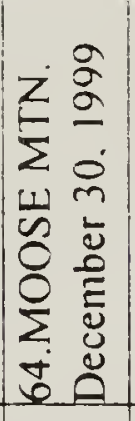 & 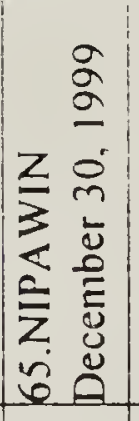 & 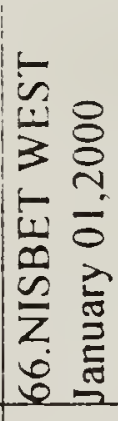 & 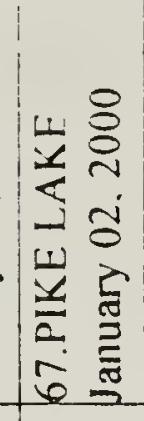 & 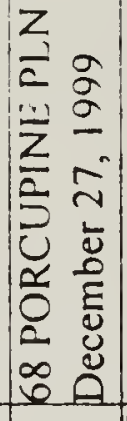 & 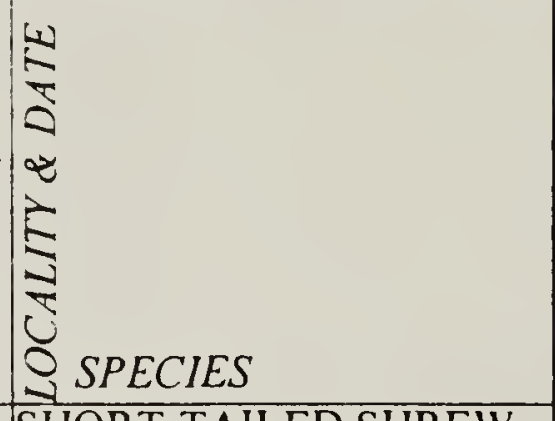 \\
\hline & & & & & & & & & & SHORT-TAILED SHREW \\
\hline & & & & & & & & & & MASKED SHREW \\
\hline & & & & & & & & & & SHREW species \\
\hline & & & & & & & & & & EASTERN COTTONTAIL \\
\hline 2 & & & & & & & & & & NUTTALL'S \\
\hline & $15 \mathrm{~T}$ & & & & & $T$ & & $2 \mathrm{I}+20 \mathrm{~T}$ & & SNOWSHOE HARE \\
\hline & & 0 & 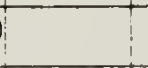 & 3 & & & & $2 T$ & & WHITE-T. JACKRABBIT \\
\hline & & & & & & & & & & RICH. GND. SQUIRREL \\
\hline & & $t$ & & & & & & & & GREY SQUIRREL \\
\hline & & & & 9 & & & & & & FOX SQUIRREL \\
\hline & 3 & & & & 5 & 3 & & 5 & & RED SQUIRREL \\
\hline & & & & & & & & & & N. FLYING SQUIRREL \\
\hline & & & & & & & & & & SQUIRREL species \\
\hline & & & & & & & & & & BEAVER \\
\hline & & & & & & & & & & DEER MOUSE \\
\hline & & & & & & & & & & GAP. RED-BACKED VOLE \\
\hline & & & & & & & & & & MUSKRAT \\
\hline & & & & & & & & & & MEADOW VOLE \\
\hline & & & & & $\overrightarrow{1}$ & & & $1 \mathrm{~T}$ & & VOLE species \\
\hline & $4 \mathrm{~T}$ & & $1 \mathrm{~T}$ & & & & & $4 \mathrm{~T}$ & & MOUSE species \\
\hline & & & & & & & & & & NORWAY RAT \\
\hline & & & & & & & & & & THOUSE MOUSE \\
\hline 3 & 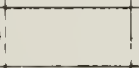 & & 1 & 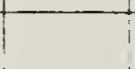 & & & & & & PORCUPINE \\
\hline 0 & $\mathrm{TT}$ & $\frac{\vec{t}}{4}$ & 0 & & & 1 & T & $\overline{3}$ & & COYOTE \\
\hline & & & & & & & & & & WOLF \\
\hline & & & & & & & & IT & & RED FOX \\
\hline & & & & & & & & & & SWIFT FOX \\
\hline & & & & & & & & & & RACOON \\
\hline & & & & & & & & & & ERMINE \\
\hline & & & & & & & & & & LONG-TAILED WEASEL \\
\hline & & & & & & & & & & LEAST WEASEL \\
\hline & $1 \mathrm{~T}$ & & & & & & & & & WEASEL species \\
\hline & & & & & & & & 1 & & MINK \\
\hline & & & & 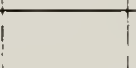 & & & 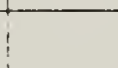 & ! & & BADGER \\
\hline & 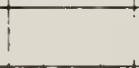 & & & & & & & & & TSTRIPED SKUNK \\
\hline & & & & & & & & & & RIVER OTTER \\
\hline 31 & & & & & & & & & & MULE DEER \\
\hline & $2+12 T$ & & 5 & & & & & 14 & & TWHITE-TAILED DEER \\
\hline 1 & 1 & & & & & & & $15+7 T$ & & DEER species \\
\hline & $2 T$ & & & & & & & & & TMOOSE \\
\hline 29 & & & & & & 31 & & & $20 \mathrm{~T}$ & TELK \\
\hline 19 & & & & & & & & & & PRONGHORN \\
\hline $\overrightarrow{7}$ & 7 & 2 & 4 & 2 & 2 & 4 & 4 & 3 & & 8TOTAL SPECIES \\
\hline
\end{tabular}


Table 1-7: SASKATCHEWAN CHRISTMAS MAMMAL COUNTS

SPECIES

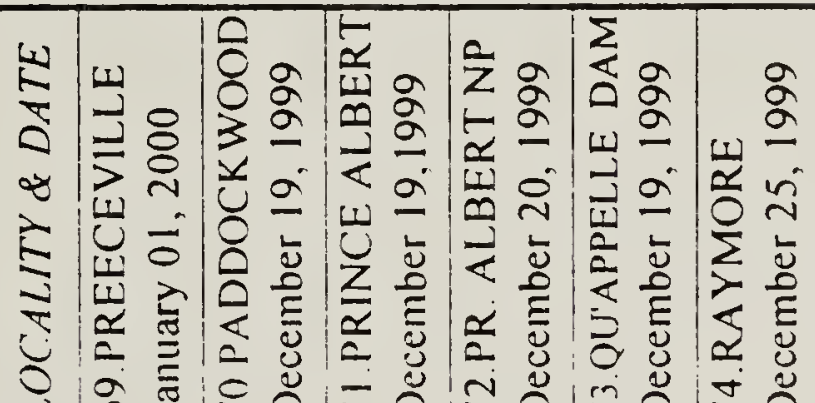

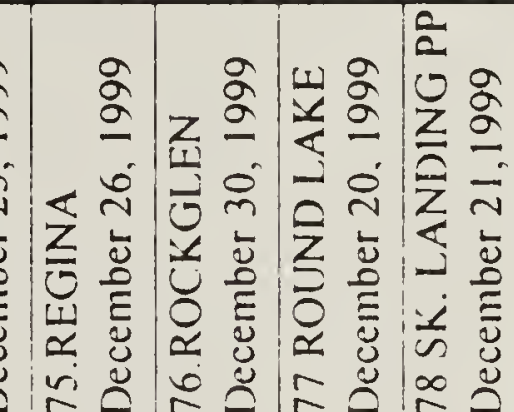
SHORT-TAILED SHREW MASKEI SHREW

SHREW species EASTERN COTTONTAIL NUTTALL'S COTTONTAIL SNOWSHOE HARE WHITE-T. JACKRABBIT RICH. GND. SQUIRREL GREY SQUIRREL FOX SQUIRREL RED SQUIRREL N. FLYING SQUIRREL SQUIRREL species BEAVER DEER MOUSE GAP. RED-BACKED VOLE MUSKRAT

MEADOW VOLE VOLE species MOUSE species NORWAY RAT HOUSE MOUSE PORCUPINE COYOTE

WOLF

RED FOX

SWIIT FOX RACOON ERMINL:

LONG-TAILED WEASEL LEAST WEASEL WEASEL species

MINK

BADGER

STRIPED SKUNK

RIVER OTTER MULE DEER WHITE-TAILED DEER DEER species MOOSE ELK PRONGHORN TOTAL SPECIES

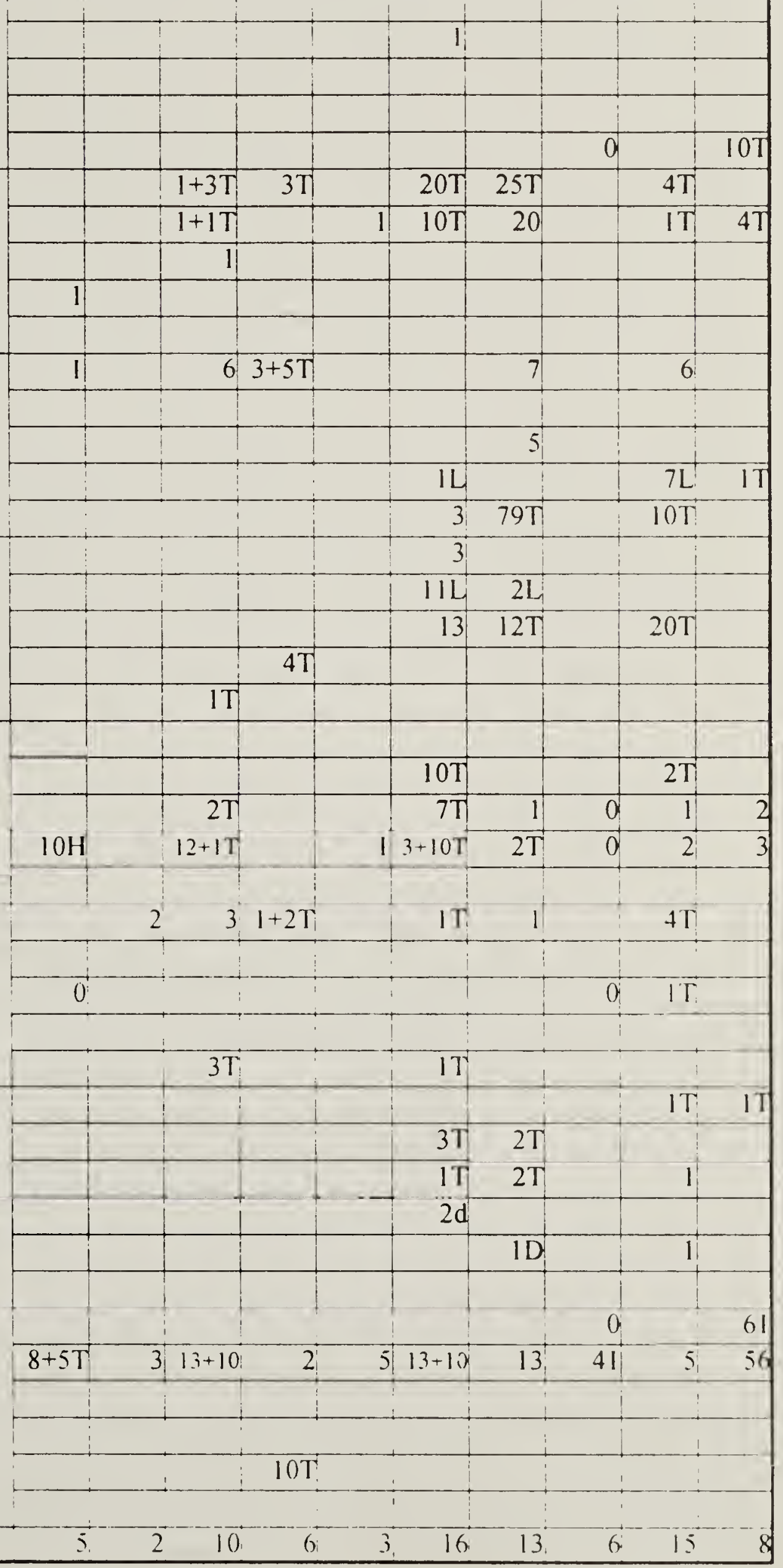




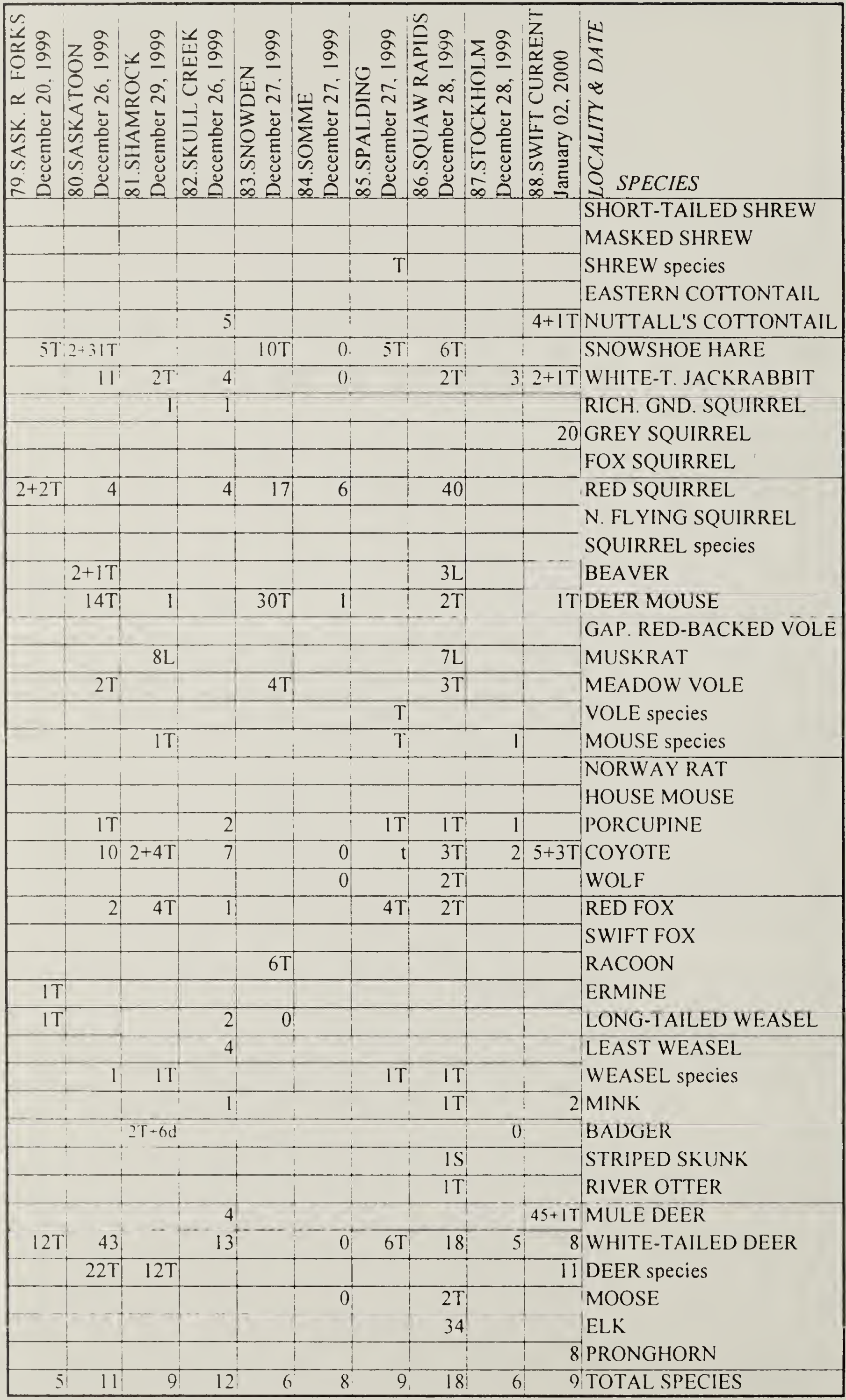




\section{SPECIES}

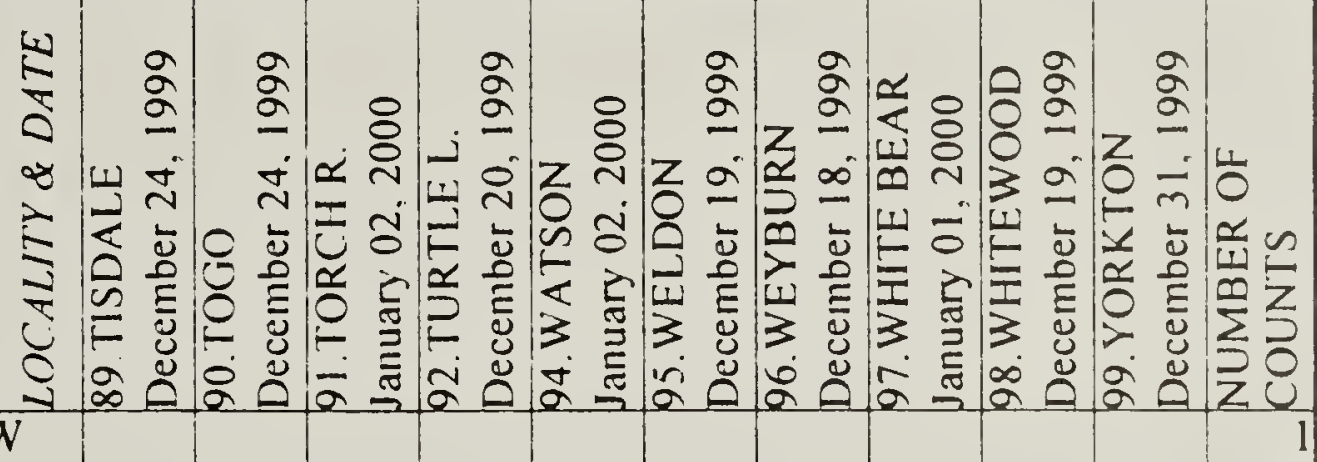
MASKED SHREW SHREW species EASTERN COTTONTAIL NUTTALL'S COTTONTAIL SNOWSHOE HARE WHITE-T. JACKRABBIT RICH. GND. SQUIRREL GREY SQUIRREL FOX SQUIRREL RED SQUIRREL N. FLYING SQUIRREL SQUIRREL species BEAVER DEER MOUSE GAP. RED-BACKED VOLE MUSKRAT MEADOW VOLE VOLE species MOUSE species NORWAY RAT HOUSE MOUSE PORCUPINE COYOTE WOLF $\overline{R E D F O X}$ SWIFT FOX RACOON ERMINE

LONG-TAILED WEASEL LEAST WEASEL WEASEL species MINK BADGER STRIPED SKUNK RIVER OTTER MULE DEER WHITE-TAILED DEER DEER species MOOSE ELK' PRONGHORN TOTAL SPECIES

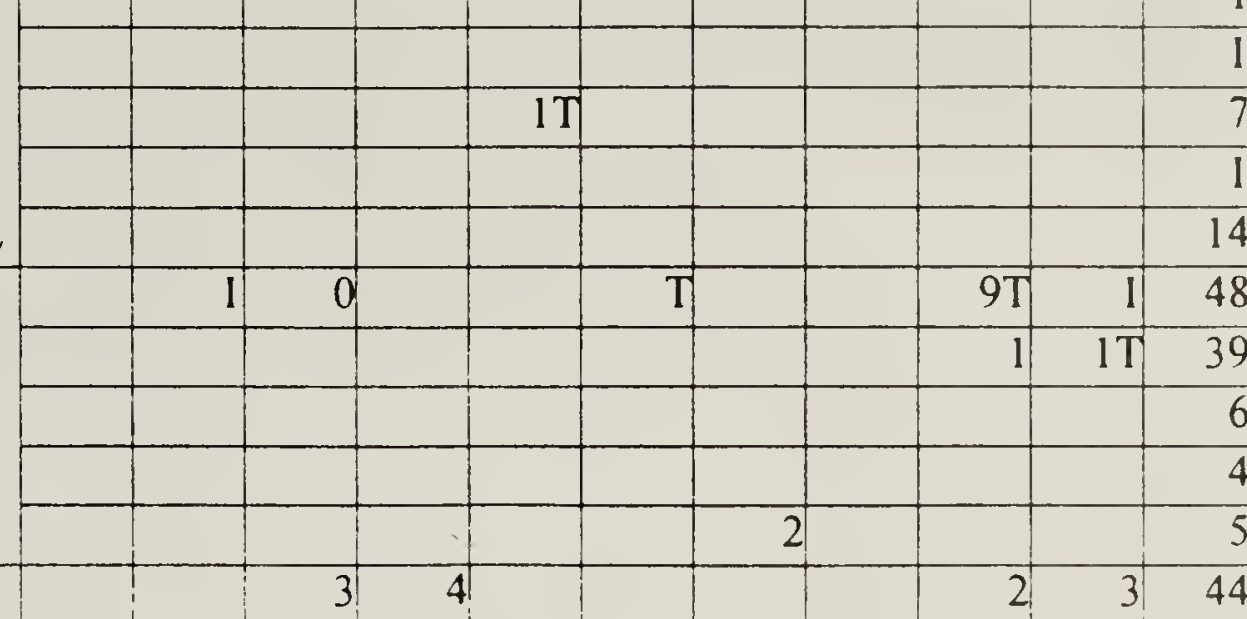

\begin{tabular}{|r|r|r|r|r|r|r|r|r|r|r|}
\hline & & & & & & & & & & 1 \\
\hline & & & & & & & & & 1 & 19 \\
\hline & & & & & & & & $2 \mathrm{~T}$ & $1 \mathrm{~T}$ & 22 \\
\hline & & & & & & & & & & 2 \\
\hline & & & & & & & & & $4 \mathrm{~T}$ & 20 \\
\hline & & & & & & & & $1 \mathrm{~T}$ & & 15 \\
\hline & & & & & & & & & $12 \mathrm{~T}$ & 21 \\
\hline & & & & & & & & & & 1 \\
\hline & & & & & & & & 1 & \\
\hline & & & 3 & & & & & 2 & 36 \\
\hline & & 0 & 7 & IT & 2 & & 6 & 2 & 76 \\
\hline & & & & & & & & & & 5 \\
\hline
\end{tabular}

\begin{tabular}{|r|r|r|r|r|r|r|r|r|r|}
\hline & & & & & & & & & 1 \\
\hline & & & & & & & & & \\
\hline
\end{tabular}

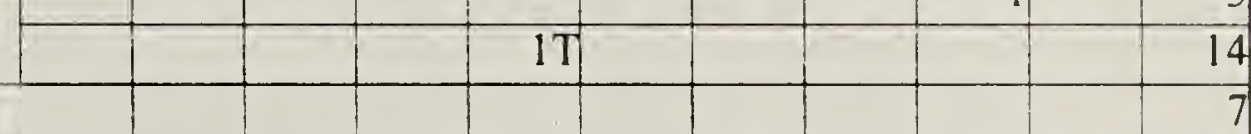

\begin{tabular}{|r|r|r|r|r|r|r|r|r|r|r|}
\hline & & & & & & & & & & 15 \\
\hline & & & & & & & & & & 15 \\
\hline
\end{tabular}

\begin{tabular}{|r|r|r|r|r|r|r|r|r|r|}
\hline & & & & & & & & & 9 \\
\hline & & & & & & & & 2 & 12 \\
\hline & & & & & & & & & 1 \\
\hline & & & 8 & & & 128 & & 24 \\
\hline & 3 & 15 & & & 15 & 3 & 5 & 11 & 77 \\
\hline
\end{tabular}

\begin{tabular}{llllllllll}
8 & 3 & 15 & & $T$ & 1 & 3 & 5 & 1 & 77 \\
\hline & & & $5 \mathrm{~T}$ & & & & & & 7
\end{tabular}




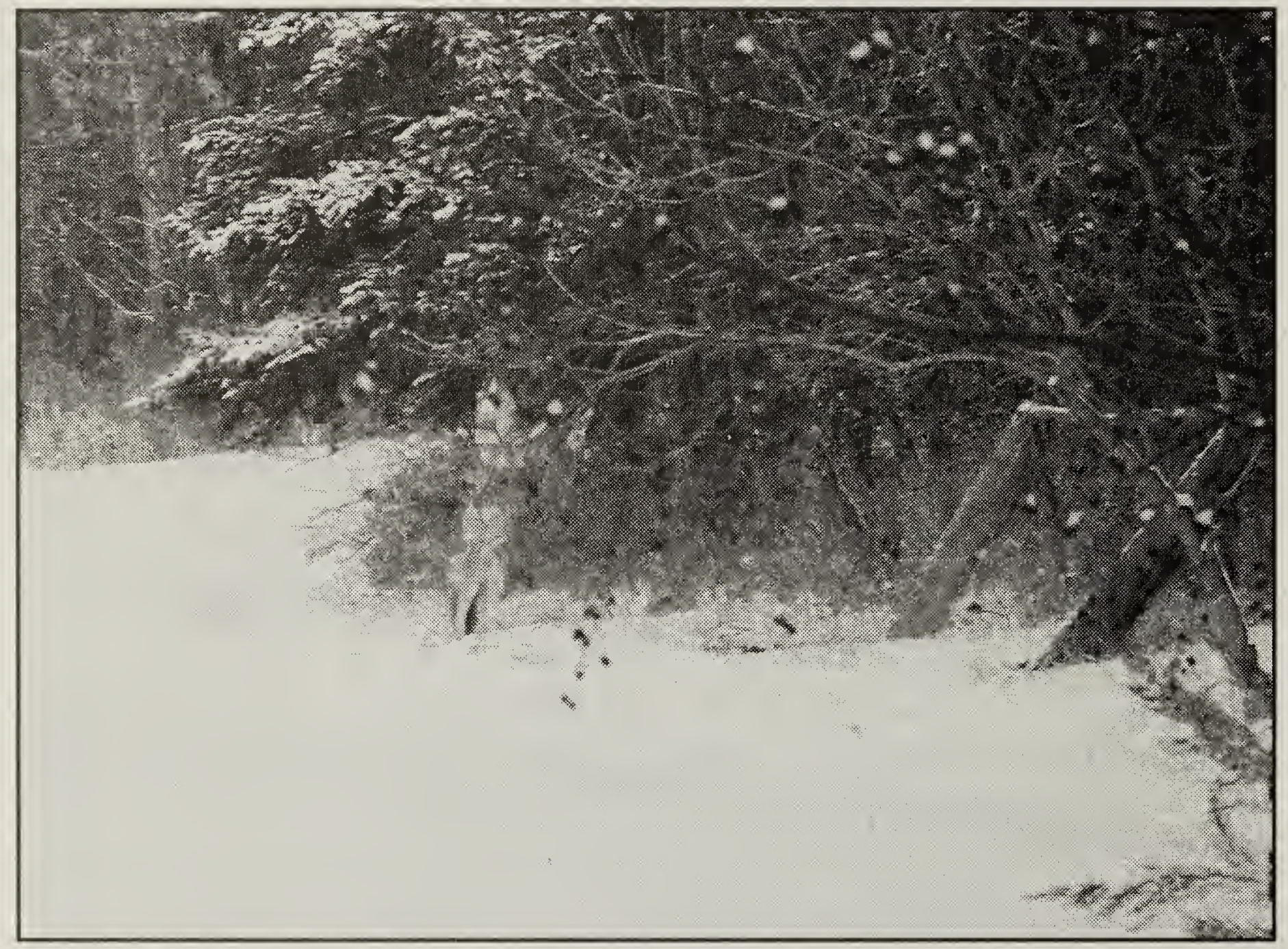

Coyote eating Crab Apples at Crooked River Joyce Mehler

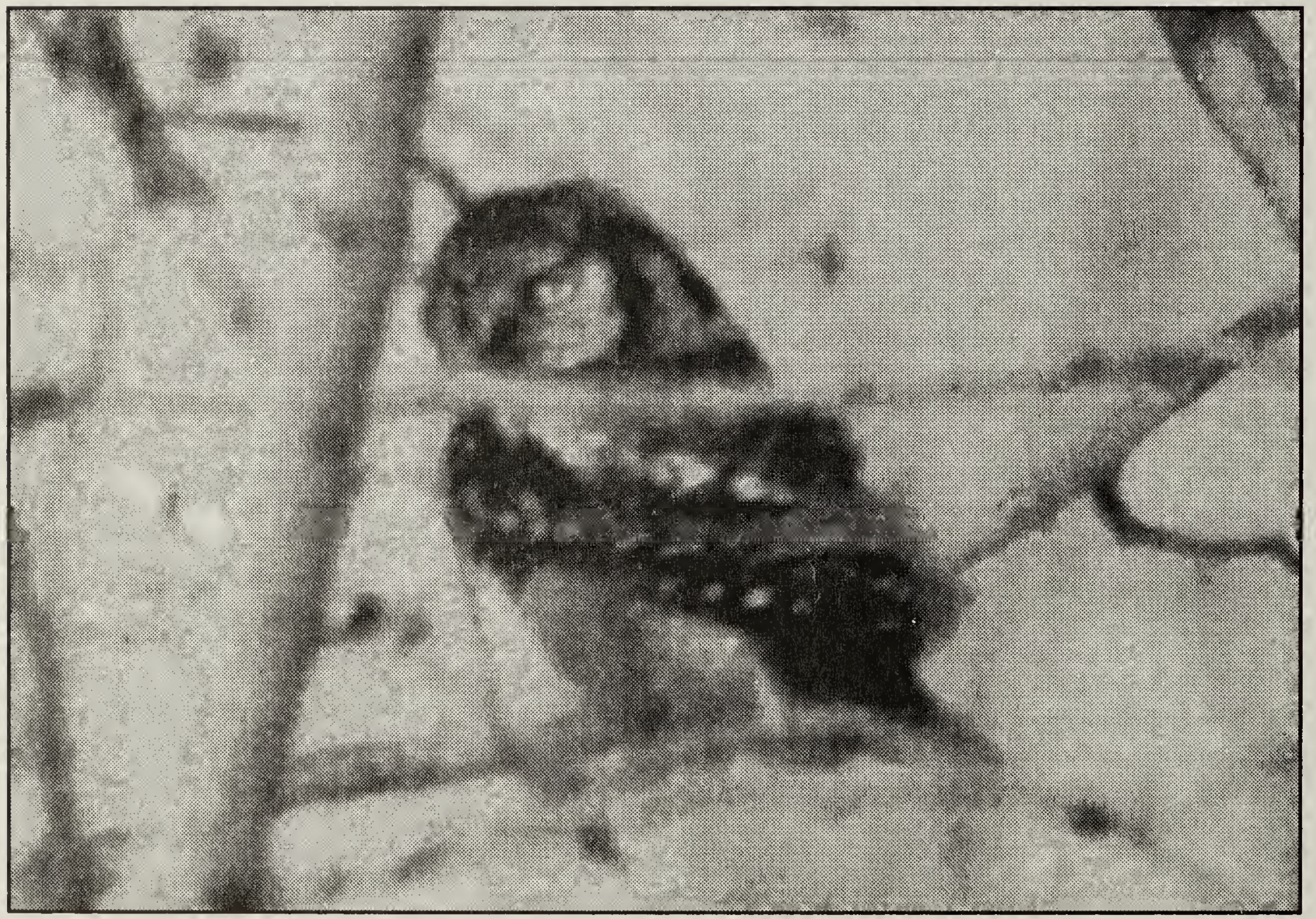

